\title{
Nanoscopic Investigation of Magnetic Thin Films by Means of Magnetic Force Microscopy Technique
}

\author{
A. SikOrA ${ }^{a}$, M. OZIMEK ${ }^{a, b, *}$ AND W. WILCZYŃSKI ${ }^{c}$ \\ ${ }^{a}$ Electrotechnical Institute Division of Electrotechnology and Materials Science, \\ M. Skłodowskiej-Curie 55/61, 55-369 Wrocław, Poland \\ ${ }^{b}$ International Laboratory of High Magnetic Fields and Low Temperatures, Gajowicka 95, 52-421 Wrocław, Poland \\ ${ }^{c}$ Electrotechnical Institute, M. Pożaryskiego 28, 04-703 Warszawa, Poland
}

(Received August 7, 2015; in final form February 29, 2016)

\begin{abstract}
In the article the results of the research aimed at the recognition of the correlation between the $\mathrm{Ni}-\mathrm{Fe}$ film thickness and its magnetic domain structures are described. Magnetic thin films were prepared by pulse magnetron sputtering. Obtained thin film thicknesses were in the range of 47.6-326.0 nm. Magnetic domain structures were imaged using magnetic force microscopy. In order to obtain quantitative description of magnetic domains images, the algorithms designed for topography parameters determination were applied, enabling the comparison of specific factors related to the magnetic properties of the samples. Utilized approach provided the analysis of the impact of sputtering parameters on the morphological and magnetic properties of obtained films.
\end{abstract}

DOI: 10.12693/APhysPolA.129.1226

PACS/topics: 81.15.Cd, 68.37.Rt, 75.70.Kw

\section{Introduction}

Magnetic thin films with a good soft magnetic properties are commonly used in the area of memory devices for computer magnetic recording media, sensor industry and microelectromechanical systems. In particular, magnetic iron-nickel alloys with 72-83\% nickel (generally called Sypermalloys, Mumetal) are well known group of thin films, because of its high initial and maximum permeabilities, magnetic saturation and relatively low coercivity $[1,2]$. One of the methods of providing high quality soft magnetic thin films is magnetron sputtering [3-7]. This technique allows to deposit films on various substrates such as: glass, metal, plastic, fabric. Moreover, by magnetron deposition, the composition of alloy films can be controlled and the adhesion of film to substrate can be improved. Those advantages were key reasons to start the development of this method.

Macroscopic properties of thin films (electrical, mechanical, optical etc.) strongly depend on various factors such as: the structure and orientation, composition, thickness, grain size, type and temperature of substrates etc. [8-15]. In addition, in the case of the magnetic thin films, their magnetic properties are conditioned by the magnetic domain structure [16-18]. In order to obtain the information about the magnetic domains structure, one can use magnetic force microscopy (MFM) [19, 20]. This method is derivative technique from intermittent contact atomic force microscopy (AFM), where the utilization of the magnetic material covering the scanning

\footnotetext{
* corresponding author; e-mail: mariusz.ozimek@ml.pan.wroc.pl
}

tip along with so called "lift mode" allows to detect long range magnetic forces and to perform the imaging of the magnetic domains distribution.

In the previous study, the research was aimed at the recognition of magnetic domain structures of thin magnetic film that can be used as a shield [21]. The utilization of certain image analysis features was presented as practical approach towards the quantitative data interpretation. In this paper the results of the investigation aimed at the analysis of the correlation of the film properties and sputtering parameters are presented. In order to do that, a number of samples had to be prepared and investigated in relation to specifically adjusted process parameters. In case of magnetic thin films, the origin of this relation is connected to size, shape and homogeneity of magnetic domains structure [22, 23].

\section{Experimental work}

The experimental work contained two major steps: the samples preparation and the measurements. Both steps were performed carefully in order to avoid the samples contamination, which could have an impact on the measurement results.

Magnetic thin films were prepared by pulse magnetron sputtering in vacuum chamber $0.2 \mathrm{~m}^{3}$ of volume, equipped with rotary and turbomolecular pumps. This system yields a base pressure in the deposition chamber better than $6 \times 10^{-4} \mathrm{~Pa}$. The experimental part was carried out using planar magnetron source of the WMK100 type. $\mathrm{Ni}_{80} \mathrm{Fe}_{20}$ target with $100 \mathrm{~mm}$ in diameter and $1 \mathrm{~mm}$ thick, was sputtered onto Si substrate at room temperature. Deposition was carried out with power $550 \mathrm{~W}$ controlled by the rectangular pulses with the frequency ranging from $100 \mathrm{~Hz}$ to $5 \mathrm{kHz}$ and different argon pressure of $3.4 \times 10^{-1} \mathrm{~Pa}$ and $7.4 \times 10^{-1} \mathrm{~Pa}$ (therefore the samples 
codes M3 and M7, respectively, were used). Obtained ferromagnetic thin film thicknesses were in $47.6-326.0 \mathrm{~nm}$ range.

The measurements of obtained films thickness and the magnetic domain structure were performed in tapping mode and MFM mode respectively with Innova instrument from Bruker (former Veeco Co.). Obtained results allowed to evaluate both: topography and magnetic properties. The measurements were performed using MESPLC probes from Bruker. The lift-mode height was set to $40 \mathrm{~nm}$, in order to obtain selective detection of the long-range magnetic forces.

In order to measure the thickness of the film, the procedure was performed as follows: every sample was scratched with copper, sharp tip being cut from the wire. Such an approach allowed to obtain clear surface of the substrate with undamaged film, therefore one could easily find the edge of metallic layer. After acquiring the topography of the step height of the material, the data was analyzed. As the first step, the local facet leveling was performed in order to obtain properly adjusted faces of the substrate and metallic film. Then the height of the film was determined using five cross-sections and the height distribution statistics (Fig. 1). It should be noted that both approaches provided the outcome with good agreement. The average standard deviation of the measurements was approximately $7 \mathrm{~nm}$.

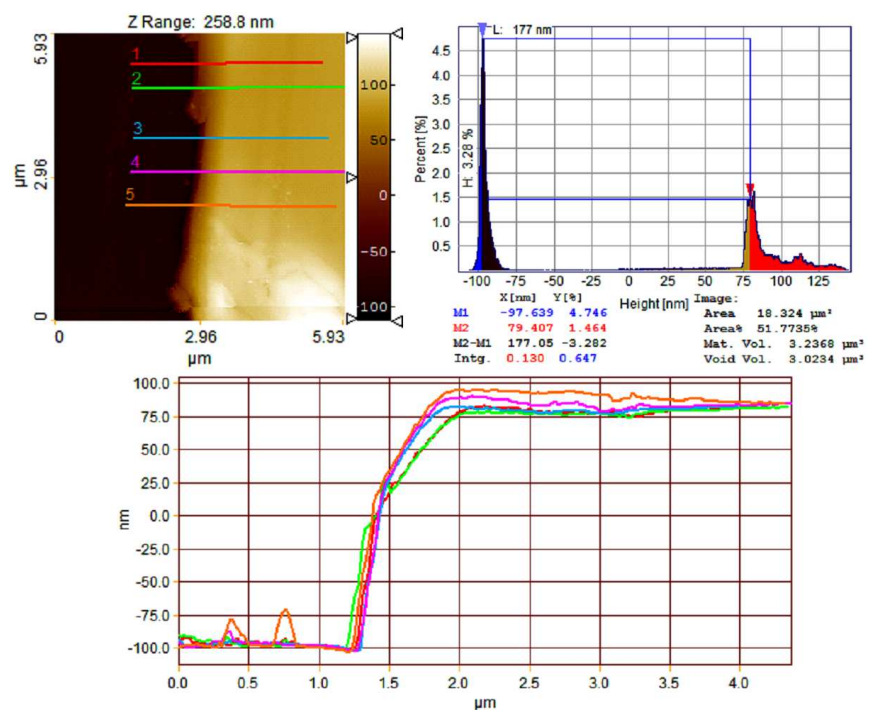

Fig. 1. The topography, surface height distribution statistics and multi cross-section of the "step" structure made of metallic film and glass substrate used for the film thickness determination.

The acquired data was processed using SPIP software [24]. The statistical parameters used in further analysis such as $S_{\mathrm{q}}$ (square mean average roughness) and $S_{\mathrm{dr}}$ (surface area ratio) are defined below:

$$
\begin{aligned}
S_{q} & =\sqrt{\frac{1}{M N}} \sum_{k=0}^{M-1} \sum_{l=0}^{N-1} z\left(x_{k}, y_{l}\right)^{2}, \\
S_{\mathrm{dr}} & =\frac{\sum_{k=0}^{M-2} \sum_{l=0}^{N-2} A_{k l}-(M-1)(N-1) \delta x \delta y}{(M-1)(N-1) \delta x \delta y} 100 \%,
\end{aligned}
$$

where

$$
\begin{aligned}
& A_{k l}=\frac{1}{4}\left[\sqrt{\delta y^{2}+\left(z\left(x_{k}, y_{l}\right)-z\left(x_{k}, y_{l+1}\right)\right)^{2}}\right. \\
& \left.+\sqrt{\delta y^{2}+\left(z\left(x_{k+1}, y_{l}\right)-z\left(x_{k+1}, y_{l+1}\right)\right)^{2}}\right] \\
& \quad \times\left[\sqrt{\delta x^{2}+\left(z\left(x_{k}, y_{l}\right)-z\left(x_{k+1}, y_{l}\right)\right]^{2}}\right. \\
& \left.\quad+\sqrt{\delta x^{2}+\left(z\left(x_{k}, y_{l+1}\right)-z\left(x_{k+1}, y_{l+1}\right)\right)^{2}}\right] .
\end{aligned}
$$

$M$ and $N$ are the total number of pixels in the analyzed scan in $x$ and $y$ axis, respectively, $k$ and $l$ are coordinates of a given pixel, $z\left(x_{k}, y_{i}\right)$ is the height of the surface at a given coordinate.

\section{Results}

Figure 2 shows the examples of the MFM images of $\mathrm{Ni}-\mathrm{Fe}$ films with different thicknesses taken in zero field at room temperature. As can be seen in Fig. 2a, for $\mathrm{a} \approx 50 \mathrm{~nm}$ thick film, there are no distinctive domain structures and domain walls. The MFM image with stronger contrast but still without clear domain structures is shown in Fig. 2b. Because the MFM tip is sensitive to field gradients from $z$-oriented domains, the obtained low contrast images imply that the magnetic spins mainly lie in the in-plane direction [16]. With further increase in film thickness, the domain structures appear and the elongated domain structure starts to be dominant. In $\approx 110 \mathrm{~nm}$ thick film, the magnetic moment of two side-neighboring domains are oriented opposite (Fig. 2c). The observed bright and dark strips arises from the magnetization canted up or down out of the plane. The stripe-like domain contrast becomes stronger as the film thickness is further increased. Figure 2d presents example of the MFM pattern obtained for the film thickness $z \approx 200 \mathrm{~nm}$.

Obtained MFM patterns clearly reveal that there is a critical film thickness to have stripe-line domains that suggests the presence of a clear out-of-plane anisotropy in prepared thin film. Such reorientation of the magnetization is accompanied by marked changes in the morphology and the arrangement of the magnetic domains.

The summarized results of the thickness measurement are shown in Fig. 3. In general, as expected, the thickness of obtained metallic films is proportional to the sputtering time.

The roughness parameter $S_{\mathrm{q}}$ revealed a significant surface roughness increase after reaching certain threshold film thickness value (Fig. 4). As in some cases the roughness is critical parameter, one can may take into account such phenomena. It should be underlined that for investigated sputtering parameters the critical film thickness 
a)

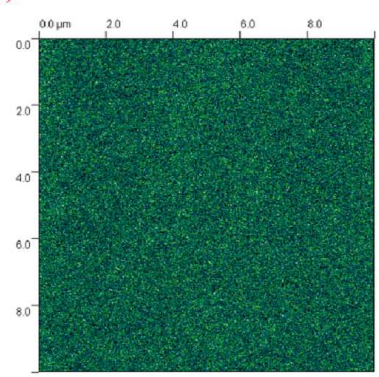

c)

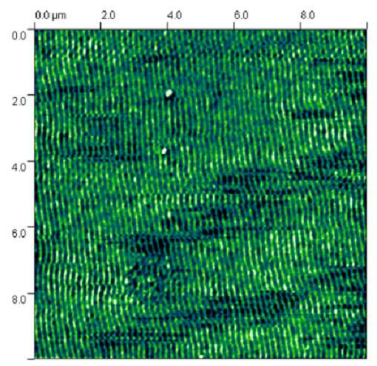

b)

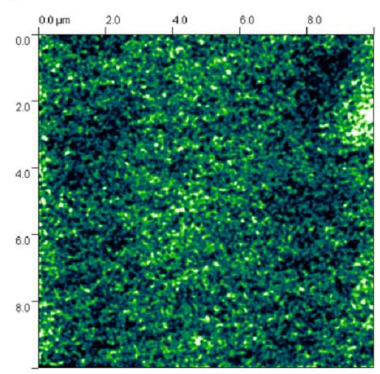

d)

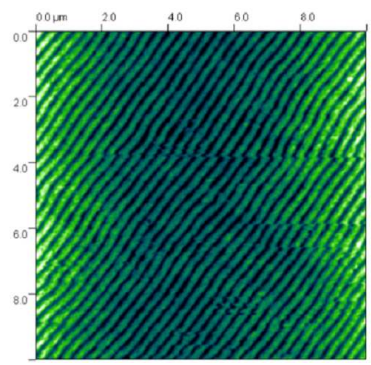

Fig. 2. MFM images of the magnetic pattern observed on the Ni-Fe thin films with different thicknesses, $z \approx$ : (a) $50 \mathrm{~nm}$, (b) $65 \mathrm{~nm}$, (c) $110 \mathrm{~nm}$, (d) $200 \mathrm{~nm}$.

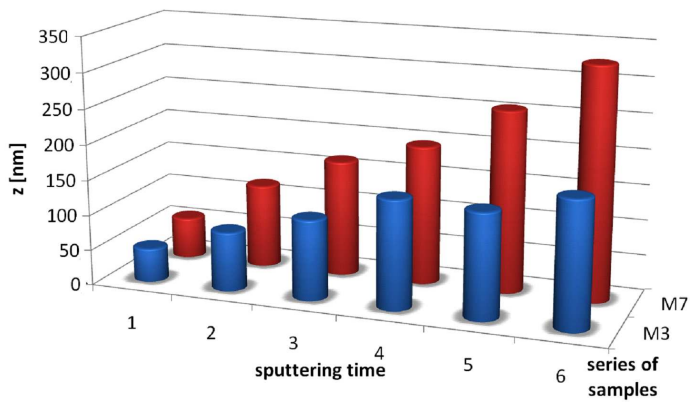

Fig. 3. The thickness of developed samples vs. sputtering time for both series of prepared samples.

values seem to be sputtering pressure dependent, but in general the behavior is similar.

Also such an issue could be observed in case of the surface area ratio (Fig. 5). One can note rapid increase of that factor for thickness above $150 \mathrm{~nm}$ and $200 \mathrm{~nm}$ for M3 and M7 samples, respectively.

When a large number of the samples is analyzed, one needs to use the values representing the specific properties of the surface in order to be able to compare them. According to performed tests [21], it appeared to be convenient to characterize magnetic properties with tools designed for topography analysis.

The surface area ratio $\left(S_{\mathrm{dr}}\right)$ shows interesting relationship to the thickness of the metallic film (Fig. 6). As the magnetic interaction intensity (peak-peak value related to the force caused by the magnetic interaction) increases along with the domains density, the $S_{\mathrm{dr}}$ value raises up. Therefore this parameter may indicate the way the magnetic domain forms, with particular recognition

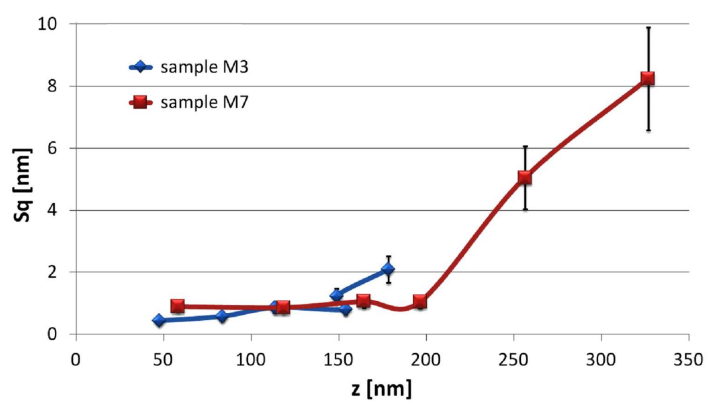

Fig. 4. The relation of the root mean square roughness $\left(S_{\mathrm{q}}\right)$ parameter vs. the thickness of the sputtered films.

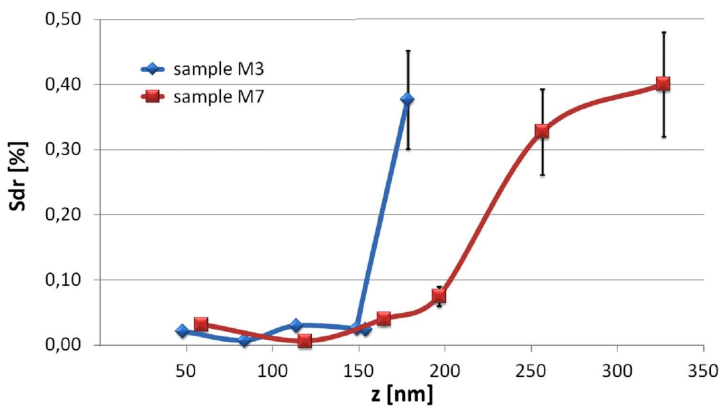

Fig. 5. The relation of the surface area ratio $\left(S_{\mathrm{dr}}\right)$ parameter vs. the thickness of the sputtered films.

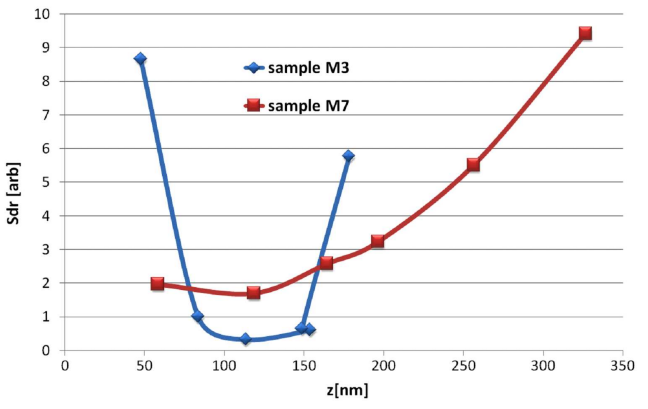

Fig. 6. The relation of the magnetic domains properties quantified using surface area ratio $\left(S_{\mathrm{dr}}\right)$ parameter vs. the thickness of the sputtered films.

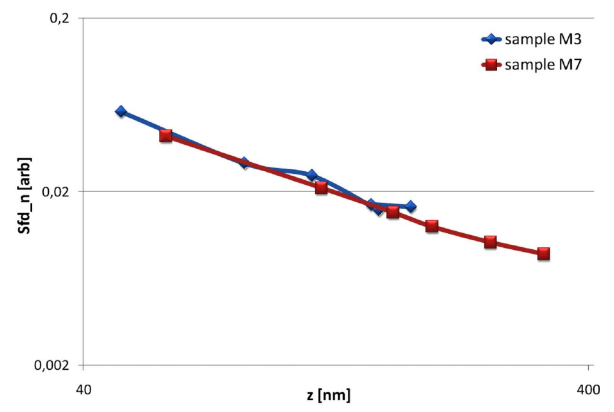

Fig. 7. The relation of the magnetic domains properties quantified using fractal dimension $\left(S_{\mathrm{fd}}\right)$ parameter vs. the thickness of the sputtered films. 
of the critical film thickness of in-plane-out-plane domains orientation, observed as local minimum at thickness approximately $110 \mathrm{~nm}$. The increase of the film thickness enabled the appearance of the laterally smaller but bigger in volume domains, causing stronger magnetic interaction and increase of $S_{\mathrm{dr}}$. The analysis of the graph allows to conclude that for both deposition rates the critical film thickness of the film is similar, but the magnetic interaction forces are different (various magnetization).

The fractal dimension is a statistical quantity that gives an indication of how completely a fractal appears to fill the space, as one zooms down to finer and finer scales. If we expect that domain structure will have very complex shape, it can be useful to describe it that way [25]. Figure 7 presents the normalized $S_{\mathrm{df}_{\mathrm{n}}}$ parameter based on fractal dimension, related to the thickness of the films. $S_{\mathrm{fd}}$ is calculated by analyzing the Fourier amplitude spectrum for the different angles. At each calculation step the amplitude Fourier profile is extracted and the logarithm of the frequency and amplitude coordinates calculated and the outcome is averaged and referred to the thickness of the investigated layer. The used formula is presented below

$$
S_{\mathrm{df}}=\frac{1}{z n} \sum_{n=0}^{180} \frac{6+s}{2},
$$

where $s$ is the slope of the log-log curves [26]. Such a data enables comparison of the intensity of the domain changes in all directions, taking into account the impact of the film thickness.

One can notice linear behavior in $\log -\log$ scale, very similar for both sputtering parameters, therefore the complexity of the magnetic domains borders increases in the same fashion for both investigated film groups.

\section{Summary and outlook}

Presented approach based on quantitative description of the MFM images allowed to analyze the impact of the sputtering factors on magnetic and morphological properties of developed films.

The major advantage of the presented analysis method is $2 \mathrm{D}$ complex and statistical outcome of the acquired data instead of simple point-to-point or profile description. Observed critical film thickness as well as the impact of the film sputtering parameters on magnetization of the domains are useful data in terms of choosing the most effective film development factors. The analysis shown here enables the possibility to perform the optimization process in terms of maximal performance as the magnetic shielding with specific focus on the oxidation risk minimizing related to the surface roughness (in particular in terms of the chemically active surface and presence of pores, which likely are filled with water and strongly increase the deterioration speed).

\section{References}

[1] C.W. Chen, Magnetism and Metallurgy of Soft Magnetic Materials, Dover, New York 1986.
[2] R.C. O'Handley, Modern Magnetic Materials: Principle and Applications, Wiley-Interscience, New York 2000.

[3] P.J. Kelly, J.W. Bradley, J. Optoelectron. Adv. Mater. 11, 1101 (2009).

[4] X.F. Wang, P. Wu, X. Li, Z.Q. Li, H.L. Bai, E.Y. Jiang, J. Magn. Magn. Mater. 312, 147 (2007).

[5] X.H. Li, Z. Yang, Mater. Sci. Eng. B 106, 41 (2004).

[6] X. Chen, H. Qiu, P. Wu, F. Wang, L. Pan, Y. Tian, Vacuum 75, 217 (2004).

[7] R. Gupta, M. Gupta, T. Gutberlet, Pramana J. Phys. 71, 1123 (2008).

[8] A. Kumar, D. Singh, D. Kaur, Surf. Coat. Technol. 203, 1596 (2009).

[9] W. Mista, J. Ziaja, A. Gubański, Vacuum 74, 293 (2004).

[10] H. Qiu, F. Wang, P. Wu, L. Pan, Y. Tian, Vacuum 66, 447 (2002).

[11] E. Vlakhov, R. Szymczak, M. Baran, K. Piotrowski, A. Szewczyk, W. Paszkowicz, L. Lobanovskii, S. Matyajasik, K. Nenkov, H. Szymczak, Acta Phys. Pol. A 115, 89 (2009).

[12] A.K. Kulkarni, L.C. Chang, Thin Solid Films 301, 17 (1997).

[13] J. Zhou, L. Tian, J. Yan, J. Wuhan Univ. Technol.Mater Sci. Ed. 23, 159 (2008).

[14] M. Xu, G. Luo, C. Chai, Z. Mai, W. Lai, Z. Wu, D. Wang, J. Cryst. Growth 212, 291 (2000).

[15] A. Mekki, N. Tabet, Acta Phys. Pol. A 125, 365 (2014).

[16] M. Liebmann, A. Schwarz, U. Kaiser, R. Wiesendanger, Phys. Rev. B 71, 104431 (2005).

[17] J. Dho, N.H. Hur, J. Magn. Magn. Mater. 318, 23 (2007).

[18] J. McCord, S. Dieter, K. Seemann, J. Magn. Magn. Mater. 271, 46 (2004).

[19] M.R. Koblischka, U. Hartmann, Ultramicroscopy $\mathbf{9 7}$, 103 (2003).

[20] C.T. Hsieh, J.Q. Liu, J.T. Lue, Appl. Surf. Sci. 252 , 1899 (2005)

[21] M. Ozimek, A. Sikora, W. Wilczyński, Przeglad Elektrotechniczny 8, 208 (2013).

[22] J. McCord, J. Westwood, IEEE Trans. Magn. 37, 1755 (2001).

[23] S. Kalinin, D.A. Bonnell, Scanning Probe Microscopy: Characterization, Nanofabrication and Device Application of Functional Materials, Eds. P.M. Vilarinho, Y. Rosenwaks, A.Y. Kingon, Springer, Netherlands 2005, p. 199.

[24] www.imagemet.com/WebHelp6/Default.htm\# RoughnessParameters/Roughness Parameters.htm, accessed 12.10.2015.

[25] I. Stoica, A.I. Barzic, C. Hulubei, D. Timpu, Microsc. Res. Technol. 76, 503 (2013).

[26] J.C. Russ, The Image Processing Handbook, 4th ed., CRC Press, Boca Raton, FL 2002. 